\title{
Uso de Bebidas Alcoólicas entre Acadêmicos da Área de Saúde
}

\author{
Alcohol Use among Healthcare Students
}

\author{
Stéphany Kétllin Mendes Oliveira \\ Árlen Almeida Duarte de Sousa ${ }^{I}$ \\ Sandra Lopes CavalcantiI \\ Maria das Graças Monte Mello Taveira II \\ Divanise Suruagy Correia ${ }^{I I}$ \\ Daniel Antunes Freitas ${ }^{I I I}$
}

\section{PALAVRAS-CHAVE}

- Bebidas Alcoólicas;

- Odontologia;

- Epidemiologia;

- Consumo de Bebidas Alcoólicas;

- Educação Médica.

\section{KEYWORDS}

- Alcoholic Beverages;

- Dentistry;

- Epidemiology;

- Alcohol Consumption;

- Medical Education.

\section{RESUMO}

Esta pesquisa descreve o consumo de bebidas alcoólicas e a presença de fatores associados entre acadêmicos de Odontologia. É um estudo transversal com 172 acadêmicos de Odontologia, cujos dados foram obtidos por meio de questionários com questões referentes às características do consumo de álcool e à existência ou não de fatores associados a este hábito. Os resultados apontam que 58,7\% dos entrevistados relataram uso de bebidas alcoólicas, sendo a maioria do sexo masculino. Esquecer problemas e sentir-se bem foram os principais fatores motivacionais relatados. Problemas familiares e a perda de objetos foram as consequências mais observadas. A relação entre sexo e consumo mostrou-se estatisticamente significante. A relação entre sexo e as variáveis relacionadas ao consumo de álcool foi significativa para "uso em eventos sociais", "fazer amigos" $e$ "sentir-se bem em eventos sociais". Foi observado consumo elevado e preocupante de bebidas alcoólicas entre os acadêmicos.

This study aimed to describe the use of alcohol and the presence of associated factors among dental students. Cross-sectional in nature, the study surveyed 172 dental students, whose data was obtained through questionnaires with questions regarding the characteristics of their alcohol consumption and the presence or absence of factors associated with this habit. The results show that $58.7 \%$ of respondents reported using alcohol, with the incidence higher among male students. The main reported motivating factors were forgetting their problems and feeling good, with the most frequently observed consequences family problems and losing belongings. The relationship between gender and consumption was statistically significant. The relationship between sex and the variables related to alcohol consumption was significant for "use in social events", "making friends" and "feeling good at social events". The overall findings suggested excessive and worrying levels of alcohol consumption among students. 


\section{INTRODUÇÃO}

O álcool é a substância psicoativa mais utilizada pela população mundial, sendo seu uso amplamente aceito e difundido por diversos povos e culturas ${ }^{1}$. Embora traga modificações de comportamento e possua grande impacto na deterioração das relações humanas, tem sido frequentemente confundido como porta de acesso à interação social ${ }^{2}$.

Seu uso pode trazer muitas consequências prejudiciais ao indivíduo e à coletividade: problemas de saúde, tanto física como psicológica, nas relações familiares, sociais e de emprego, acidentes domésticos, do trabalho e de trânsito, comportamento violento e automutilação; também está relacionado ao desenvolvimento de epidemias, como acidente vascular cerebral, câncer e absenteísmo escolar, problemas interpessoais e comportamentos sexuais de risco ${ }^{3}$. O consumo de álcool é também um fator de risco na gravidez e para a gravidez indesejada, estado nutricional e dieta ${ }^{4}$.

Para os jovens, o álcool tem o poder de aumentar as potencialidades físicas e sociais, dando-lhes coragem e confiança não usuais ${ }^{5}$. Em verdade, isto é um mito que, de tão presente no âmbito social, acaba sendo transmitido de geração em geração e se transformandonuma prática cotidiana juvenil ${ }^{6}$.

Geralmente, os indivíduos começam a ingestão de álcool no período da adolescência, permanecendo na sua juventude e prolongando pela fase adulta ${ }^{7}$. Isto ocorre, em vários casos, devido à necessidade de socialização nesta fase de transição para a vida adulta ${ }^{8}$. Assim, não percebem que aos poucos estão ingerindo álcool em excesso, hábito que pode acarretar consequências futuras ${ }^{7}$. Estudos realizados entre estudantes universitários demonstram que o índice de consumo de álcool é muito significativo ${ }^{3,8-10}$.

O objetivo deste estudo foi descrever o consumo de bebidas alcoólicas ea presença de fatores associados entre acadêmicos de Odontologia de umainstituição de ensino superior, situada na região norte de Minas Gerais.

\section{METODOLOGIA}

Trata-se de um estudo de natureza exploratória, descritiva e transversal, com abordagem metodológica quantitativa.

O grupo pesquisado era composto por estudantes de graduação em Odontologia de uma instituição de ensino superior privada, situada em Montes Claros, norte de Minas Gerais. Foram entrevistados todos os acadêmicos que concordaram em participar da pesquisa, tendo sido excluídos os que não estavam devidamente matriculados, frequentes ou que não fossem encontrados em três tentativas. Responderam ao questionário 172 acadêmicos de um total de 197.
Os dados foram coletados entre os meses de fevereiro e abril de 2014, por acadêmicas jovens-pesquisadoras do Programa de Iniciação Científica da própria instituição, capacitadas e treinadas para a aplicação dos questionários e o esclarecimento de eventuais dúvidas dos participantes. O trabalho de campo foi orientado e supervisionado pelo coordenador-geral do projeto. O questionário utilizado foi adaptado do instrumento usado pelo Departamento de Odontologia Social da Universidade de Cartagena (Colômbia) ${ }^{11}$. Trata-se de um questionário autoaplicável, sem identificação nominal, cujas questões são de fácil assimilação para o grau de conhecimento do grupo pesquisado. Os questionários foram aplicados durante as atividades acadêmicas.

O Programa Statistical Package for the Social Sciences (SPSS) 18.0 para Windows foi utilizado para análise estatística. A variável dependente do estudo foi o uso de álcool, classificada dicotomicamente (sim/não). Deu-se a análise e interpretação dos resultados por análises descritivas e cálculo dos intervalos de confiança (IC 95\%). O teste do qui-quadrado de Pearson e Odds Ratio(OR) foram empregados para avaliar a força de associação entre as variáveis independentes e a variável dependente $(\mathrm{p}<0,05)$.

As questões éticas foram devidamente observadas. A pesquisa foi aprovada pelo Comitê de Ética em Pesquisa da Associação Educativa do Brasil-Soebras com o Protocolo 01622/11 e todos os entrevistados assinaram o Termo de Consentimento Livre e Esclarecido.

\section{RESULTADOS}

O presente estudo compreendeu 172 participantes com idades entre 18 e 32 anos (média=23,6; DP=5,2). A maioria dos acadêmicos entrevistados era do sexo feminino $(58,1 \%)$, sendo que $43,6 \%$ cursavam os períodos iniciais (primeiro ao terceiro período); $29,1 \%$ os períodos intermediários (quarto ao sexto período); e 27,3\% os períodos finais (sétimo ao décimo período). Cento e um acadêmicos (58,7\%), entre os 172 entrevistados, informaram consumir bebidas alcoólicas.

Entre os participantes que informaram consumir bebidas alcoólicas, a maior parte era do sexo masculino (52,5\%); 40,6\% estavam cursando os períodos iniciais; $52,5 \%$ relataram que o consumo ocorria em momentos distintos de eventos sociais; $54,5 \%$ com frequência superior a três vezes por mês; e 78,2\% informaram não levar em consideração o fato de estar ou não em período escolar.

A Tabela 1 mostra, entre os acadêmicos que relataram consumir bebidas alcoólicas,os fatores motivacionais que os levaram a este consumo. 


\section{TABELA 1}

Fatores motivacionais para o consumo de álcool entre estudantes de Odontologia. Montes Claros, MG, Brasil, 2014 (n=101)

\begin{tabular}{|c|c|c|c|}
\hline Variáveis & n & $\%$ & *IC 95\% \\
\hline \multicolumn{4}{|l|}{ Amigos consomem } \\
\hline Sim & 12 & 11,9 & $(5,6 ; 18,2)$ \\
\hline Não & 89 & 88,1 & $(81,8 ; 94,4)$ \\
\hline \multicolumn{4}{|l|}{ Estar na moda } \\
\hline Sim & - & - & - \\
\hline Não & 101 & 100,0 & - \\
\hline \multicolumn{4}{|l|}{ Ser aceito socialmente } \\
\hline Sim & - & - & - \\
\hline Não & 101 & 100,0 & - \\
\hline \multicolumn{4}{|c|}{ Aceitação familiar do consumo } \\
\hline Sim & 12 & 11,9 & $(5,6 ; 18,2)$ \\
\hline Não & 89 & 88,1 & $(81,8 ; 94,4)$ \\
\hline \multicolumn{4}{|l|}{ Fazer amigos } \\
\hline Sim & 08 & 7,9 & $(2,6 ; 13,2)$ \\
\hline Não & 93 & 92,1 & $(86,8 ; 97,4)$ \\
\hline \multicolumn{4}{|l|}{ Ter relações sexuais } \\
\hline Sim & 1 & 1,0 & $(-0,9 ; 2,9)$ \\
\hline Não & 100 & 99,0 & $(71,1 ; 100,9)$ \\
\hline \multicolumn{4}{|l|}{ Esquecer problemas } \\
\hline Sim & 29 & 28,7 & $(19,9 ; 37,5)$ \\
\hline Não & 72 & 71,3 & $(62,5 ; 80,1)$ \\
\hline \multicolumn{4}{|l|}{ Sentir-se bem em eventos } \\
\hline Sim & 45 & 44,6 & $(36,7 ; 52,5)$ \\
\hline Não & 56 & 55,4 & $(47,5 ; 63,3)$ \\
\hline
\end{tabular}

*Foi adotado Intervalo de Confiança de 95\%.

Fonte: Dados do estudo, 2014.

Os acadêmicos que consomem bebidas alcoólicas informaram diversos tipos de consequências advindas deste consumo, conforme apresentado na Tabela 2.

A Tabela 3 apresenta os resultados obtidos no teste qui-quadrado realizado para verificar a associação da variável dependente (consumo de álcool) com as variáveis independentes (sexo e período). Encontrou-se associação entre consumo de álcool e sexo ( $\mathrm{p}=0,001)$; não houve significância relativa ao período cursado $(\mathrm{p}=0,592)$.

A Tabela 4 apresenta os resultados do teste qui-quadrado para verificar a associação entre a variável sexo e as demais variáveis com relação ao consumo de álcool. Foi significativo para as variáveis: consumo em eventos sociais $(p=0,004)$, consumo com o objetivo de fazer amigos $(p=0,039)$ e consumo para sentir-se bem $(p=0,031)$
TABELA 2

Consequências do consumo de álcool entre estudantes de Odontologia.

\section{Montes Claros, MG, Brasil, 2014 (n=101)}

\begin{tabular}{|c|c|c|c|}
\hline Variáveis & n & $\%$ & ${ }^{*} \mathrm{IC} 95 \%$ \\
\hline \multicolumn{4}{|l|}{ Problemas familiares } \\
\hline Sim & 15 & 14,9 & $(8,0 ; 21,8)$ \\
\hline Não & 86 & 85,1 & $(78,2 ; 92,0)$ \\
\hline \multicolumn{4}{|c|}{ Problemas no meio acadêmico } \\
\hline Sim & - & - & - \\
\hline Não & 101 & 100,0 & - \\
\hline \multicolumn{4}{|c|}{$\begin{array}{l}\text { Problemas de relacionamento } \\
\text { afetivo }\end{array}$} \\
\hline Sim & 4 & 4,0 & $(0,2 ; 7,8)$ \\
\hline Não & 97 & 96,0 & $(92,8 ; 99,8)$ \\
\hline \multicolumn{4}{|l|}{ Perdas de objetos } \\
\hline Sim & 17 & 16,8 & $(8,8 ; 24,8)$ \\
\hline Não & 84 & 83,2 & $(75,2 ; 91,2)$ \\
\hline \multicolumn{4}{|l|}{ Confrontos físicos } \\
\hline Sim & 5 & 5,0 & $(0,7 ; 9,3)$ \\
\hline Não & 96 & 95,0 & $(90,7 ; 99,3)$ \\
\hline \multicolumn{4}{|c|}{ Relações sexuais indesejadas } \\
\hline Sim & 5 & 5,0 & $(0,7 ; 9,3)$ \\
\hline Não & 96 & 95,0 & $(90,7 ; 99,3)$ \\
\hline \multicolumn{4}{|l|}{ Acidentes } \\
\hline Sim & 8 & 7,9 & $(2,6 ; 13,2)$ \\
\hline Não & 93 & 92,1 & $(86,8 ; 97,4)$ \\
\hline \multicolumn{4}{|l|}{ Intoxicações alcoólicas } \\
\hline Sim & 2 & 2,0 & $(-0,7 ; 4,7)$ \\
\hline Não & 99 & 98,0 & $(95,3 ; 100,7)$ \\
\hline
\end{tabular}

*Foi adotado Intervalo de Confiança de 95\%.

Fonte: Dados do estudo, 2014.

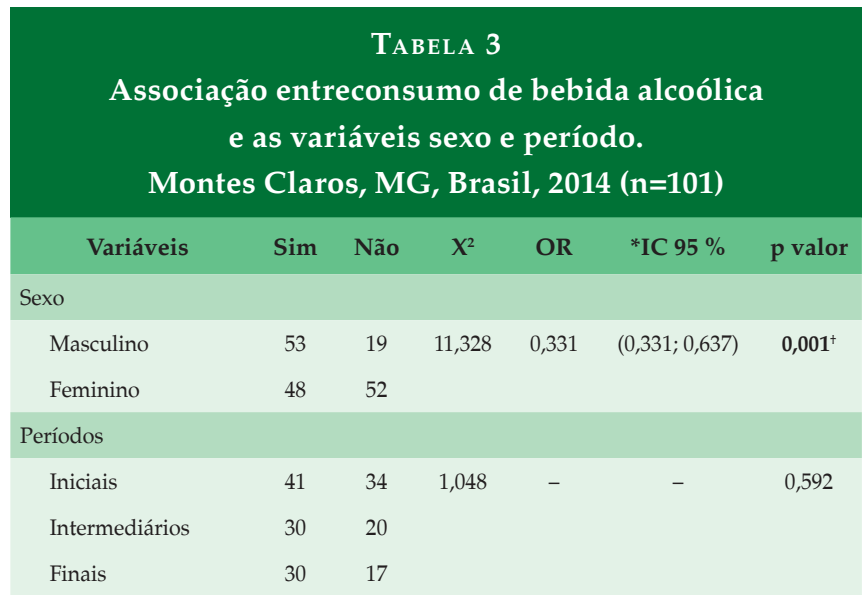

*Foi adotado Intervalo de Confiança de 95\%.

${ }^{+}$Associações foram consideradas significantes quando $p<0,05$.

Fonte: Dados do estudo, 2014. 


\begin{tabular}{|c|c|c|c|c|c|c|}
\hline \multicolumn{7}{|c|}{$\begin{array}{l}\text { Associação entre sexo e as variáveis relacionad } \\
\text { consumo de álcool. }\end{array}$} \\
\hline Variáveis & Masculino & Feminino & $\mathbf{X}^{2}$ & OR & ${ }^{*} \mathrm{IC} 95 \%$ & p valor \\
\hline \multicolumn{7}{|c|}{ Uso em eventos sociais } \\
\hline Sim & 18 & 30 & 8,225 & 0,309 & $(0,137 ; 0,697)$ & $0,004^{+}$ \\
\hline Não & 35 & 18 & & & & \\
\hline \multicolumn{7}{|c|}{ Amigos consomem } \\
\hline Sim & 6 & 6 & 0,333 & 0,894 & $(0,268 ; 2984)$ & 0,855 \\
\hline Não & 47 & 42 & & & & \\
\hline \multicolumn{7}{|c|}{ Problemas familiares } \\
\hline Sim & 9 & 6 & 0,400 & 1,432 & $(0,469 ; 4,372)$ & 0,527 \\
\hline Não & 4 & 42 & & & & \\
\hline \multicolumn{7}{|c|}{ Problemas no relacionamento } \\
\hline Sim & 3 & 1 & 0,847 & 2,820 & $(0,283 ; 28,069)$ & 0,357 \\
\hline Não & 50 & 47 & & & & \\
\hline \multicolumn{7}{|c|}{ Relações sexuais indesejadas } \\
\hline Sim & 4 & 1 & 1,598 & 3,837 & $(0,414 ; 35,594)$ & 0,206 \\
\hline Não & 49 & 47 & & & & \\
\hline \multicolumn{7}{|l|}{ Acidentes } \\
\hline Sim & 4 & 4 & 0,021 & 0,898 & $(0,212 ; 3,807)$ & 0,884 \\
\hline Não & 49 & 44 & & & & \\
\hline \multicolumn{7}{|c|}{ Fazer amigos } \\
\hline Sim & 7 & 1 & 4,274 & 7,152 & $(0,846 ; 60,447)$ & $0,039^{+}$ \\
\hline Não & 46 & 47 & & & & \\
\hline \multicolumn{7}{|c|}{ Ter relações sexuais } \\
\hline $\operatorname{Sim}$ & 1 & 00 & 0,915 & 0,981 & $(0,945 ; 1,018)$ & 0,339 \\
\hline Não & 52 & 48 & & & & \\
\hline \multicolumn{7}{|c|}{ Esquecer problemas } \\
\hline Sim & 18 & 11 & 1,501 & 1,730 & $(0,717 ; 4,175)$ & 0,220 \\
\hline Não & 35 & 37 & & & & \\
\hline \multicolumn{7}{|c|}{ Sentir-se bem em eventos } \\
\hline Sim & 29 & 16 & 4,662 & 2,417 & $(1,077 ; 5,421)$ & $0,031^{+}$ \\
\hline Não & 24 & 32 & & & & \\
\hline
\end{tabular}

*Foi adotado Intervalo de Confiança de 95\%.

${ }^{\dagger}$ Associações foram consideradas significantes quando $p<0,05$.

Fonte: Dados do estudo, 2014.

\section{DISCUSSÃO}

Na população estudada, as mulheres são maioria entre os estudantes de Odontologia; resultados semelhantes foram encontrados numa universidade colombiana e em outra instituição de ensino do Brasil ${ }^{11-12}$. Assim como em pesquisas realizadas em outras regiões brasileiras, também se observou alto número de estudantes universitários fazendo uso de bebidas alcoólicas ${ }^{3,6,14}$.Nota-se que os homens consomem mais bebidas alcoólicas do que as mulheres, resultados idênticos aos deoutros estudos publicados na literatura mundial ${ }^{12-15}$.

Assim como outros estudantes de Odontologia ${ }^{11-12}$, o grupo pesquisado também demonstrou uso frequente de bebidas alcoólicas, com a maioria relatando o consumo mais de três vezes por semana. O relato quanto ao uso independentemente de estar ou não em período de atividades escolares ressalta o alerta de que o consumo de álcool pode trazer consequências danosas ao desempenho estudantil ${ }^{6,14,16}$.Os dados referentes aos acadêmicos de Odontologia da presente pesquisa remetem a um consumo muito frequente e indiscriminado,em que não há diferenciação entre semana e fim de semana ou entre férias e aulas. É preciso chamar a atenção para esta condição, especialmente porque pesquisadores têm relacionado o consumo de álcool ao uso de drogas ilícitas ${ }^{2,14}$.

Uma parte do grupo pesquisado liga o consumo de bebidas alcoólicas ao desejo de esquecer seus problemas e também busca aplicar esta relação ao desejo de se sentir bem nos eventos sociais. Obviamente, este poder do álcool é bem conhecido: aliviar o estresse e "socializar". Mas cabe uma ressalva importante: o mesmo álcool que parece ser um remédio para os males aqui descritos, na realidade, torna-se o causador de graves problemas quando não há controle da quantidade e frequência desse consumo. Esse desejo de relaxar, deixar os problemas de lado, ficar mais tranquilo nos eventos sociaisé uma informação presente em vários estudos realizados com estudantes das profissões de saúde ${ }^{13,15,17,18}$. Esta realidade fomenta a necessidade de compreender que dificuldades são essas que podem ser debeladas pelo consumo de álcool. E pode-se indagar se essa realidade acompanhará esses indivíduos após a conclusão de sua formação acadêmica. Embora muitos não relatem a aceitação familiar como fator motivacional para o consumo de álcool, é sabido que as famílias são ambientes extremamente propícios ao desenvolvimento desse hábito; e esta aceitação pode apresentar-se como um incentivo ao início do uso de bebidas alcoólicas ${ }^{4,7,19}$.

Chama a atenção, entre os resultados, o fato de cinco estudantes do sexo feminino relatarem experiências sexuais indesejadas ligadas ao consumo de álcool. Embora, numa mensuração percentual, possa parecer um pequeno número, este dado é alarmante quando se pensa no forte impacto pessoal e coletivo decorrente de práticas sexuais sem a devida consciência. Gravidez indesejada e doenças sexualmente transmissíveis são questões que afetam, sobremaneira, o indivíduo, a família e a sociedade. Alguns estudos também mostram estudantes tendo relações sexuais motivados pelo uso de bebida alcoólica $^{7,11,18}$

Ao contrário do que foi aferido em outros grupos estudantis $2,11,13,15,19$, as respostas encontradas na presente pesquisa não são representativas para ocorrências usualmente ligadas ao álcool, como problemas no meio acadêmico e relacionamento familiar, acidentes, confrontos físicos e perda de objetos. 
Assim como em diversos estudos ${ }^{3,11-15}$ sobre consumo de álcool entre estudantes universitários em variadas partes do mundo, também neste estudo ficou evidente a alta significância do gênero em relação ao hábito: o consumo de bebidas alcoólicas é sempre maior no grupo masculino.

Já está bem estabelecido na literatura científica mundial que o consumo de álcool tem trazido extensos danos individuais e coletivos, sendo que o não uso pode ser considerado dificultador de inclusão em determinados grupos sociais. Ao beber em eventos sociais, buscando construir amizades e com o desejo de sentir-se bem, este jovem acaba por fortalecer as relações excludentes dos não bebedores ${ }^{20}$. Assim, cada vez mais estudantes aderem ao consumo de álcool com o claro intuito de não serem rejeitados.

\section{CONCLUSÕES}

Os resultados demonstram que é grande o consumo de bebidas alcoólicas entre os universitários que participaram do estudo. Este hábito é mais prevalente entre os sujeitos do sexo masculino, com alta frequência semanal, uso indiscriminado quanto ao período do consumo, sem significância quanto ao período estudantil.

Observa-se a existência de fatores motivacionais para o consumo de álcool, bem como é possível destacar a referência às consequências decorrentes deste consumo.

Os fatores sociais encontram-se, destacadamente, associados ao consumo de bebidas alcoólicas.

É fundamental a percepção de que este é um grave problema de saúde pública e que esta situação pode impactar de forma grave a sociedade, pois os acadêmicos de hoje serão os profissionais futuros que, em tese, deveriam orientar as pessoas no combate ao consumo excessivo de bebidas alcoólicas.

Se os jovens precisam beber para se sentirem acolhidos em seus grupos, esta situação se perpetuará indefinidamente. É preciso um trabalho eficiente e uma adequada abordagem desta questão para romper com esse círculo vicioso que tanto mal faz aos jovens acadêmicos.

\section{REFERÊNCIAS}

1. Galduróz JC, Noto AR, Nappo AS, Carlini EA. Trends in drug use among students in Brazil: analysis of four surveys in 1987, 1989, 1993 and 1997. Braz J Med Biol Research. 2004;37(4):523-31.

2. Lucas ACS, Parente RCP, Picanço NS, Conceição DA, Costa KRC, Magalhães IRS, et al. Use of psychoactive drugs by health sciences undergraduate students at the Federal University in Amazonas, Brazil. Cad Saúde Pública. 2006;22(3):663-71.
3. Boland M, Fitzpatrick P, Scallan E, Daly L, Herity B, Horgan J, et al. Trends in medical student use of tobacco, alcohol and drugs in an Irish university, 1973-2002. Drug Alcohol Depend. 2006;85(2):123-8.

4. Veloso LUP, Monteiro CFS. Prevalence and factors associated with alcohol use among pregnant adolescents. Rev Latino-Am Enfermagem. 2013;21(1):433-41.

5. Ferreira MMSRS, Torgal MCLFPR. Tobacco and Alcohol Consumption among Adolescents. Rev Latino-Am Enfermagem. 2010;18(2):255-61.

6. Choi D, Tolova V, Socha E, Samenow CP. Substance use and attitudes on professional conduct among medical students: a single-institution study. Acad Psychiatry. 2013;37(3):191-5.

7. Galduróz JCF, Caetano R. Epidemiology of alcohol use in Brazil. Ver Bras Psiquiatr. 2004;26(Supl I):3-6.

8. Acosta LD, Fernández AR, Pillon SC. Social risk factors for alcohol use among adolescents and youth. Rev Latino-Am Enfermagem. 2011;19(Spe):771-81.

9. MartinezRodriguez G, Luis MAV. Descriptive study of drugs use among adolescents in higher middle education in Monterrey, Nueva León, Mexico. Rev Latino-Am Enfermagem. 2004;12(Spe):391-7.

10. Bendtsen P, Johansson K, Akerlind I. Feasibility of an email-based electronic screening and brief intervention (e-SBI) to college students in Sweden. Add Behav. 2006;31(5):777-87.

11. Tovar LLA, Caballero, AJD, Martinez, GDG, Pallares, MAS. Consumo de bebidas alcohólicas y factores relacionados enestudiantes de odontología. Rev Clin Med Fam. 2001; 3(2):93-8.

12. Teixeira RF, Souza RS, Buaiz V, Marluce Siqueira MM. Uso de substâncias psicoativas entre estudantes de odontologia da Universidade Federal do Espírito Santo. Ciênc. Saúde Coletiva. 2010;15(3): 655-662.

13. Matute RC, Pillon SC. Alcohol consumption by nursing students in Honduras. Ver Lat Am Enfermagem. 2008;16(5):84-9.

14. Silva LVR, MalbergierI A, Stempliuk VA, Andrade AG. Fatores associados ao consumo de álcool e drogas entre estudantes universitários. Rev Saúde Pública. 2006;40(2): 280-8.

15. Frank E, Elon L, Naimi T, Brewer R. Alcohol consumption and alcohol counseling behaviour among US medical students: cohort study. BMJ. 2008;337:a2155.

16. Baer JS. Student factors: understanding individual variation in college drinking. J Stud Alcohol Supp. 2002;(14):40-53.

17. Kisic-Tepavcevic D, Gazibara T, Popovic A, Trajkovic G, Pekmezovic T. The impact of alcohol on health-related 
quality of life in belgrade university students. Am J Drug Alcohol Abuse. 2013;39(2):130-5.

18. Shek DT, Cheung BP. Developmental issues of university students in Hong Kong. Int J Adolesc Med Health. 2013;27:1-7.

19. Ketoja J, Svidkovski AS, Heinälä P, Seppä K. Risky drinking and its detection among medical students. Addict Behav. 2013;38(5):2115-8.

20. Baus J, Kupek E, Pires M. Prevalence and risk factors associated with drug use among school students, Brazil. Rev Saúde Pública. 2002;36(1):40-6.

\section{CONFLITO DE INTERESSES}

Não há conflito de interesses.

\section{CONTRIBUIÇÃO DOS AUTORES}

Daniel Antunes Freitas orientou a elaboração do projeto, a coleta de dados, a análise dos resultados e participou da redação do artigo; Árlen Almeida Duarte de Sousa e Stéphany Kétllin Mendes Oliveira participaram na elaboração do projeto, na coleta de dados, na análise dos resultados e na redação do artigo; Sandra Lopes Cavalcanti, Maria das Graças Monte Mello Taveira e Divanise Suruagy Correia participaram na análise dos resultados e na redação do artigo.

\section{ENDEREÇO PARA CORRESPONDÊNCIA}

Daniel Antunes Freitas.

Universidade Estadual de Montes Claros. Departamento de Saúde Mental e Saúde Coletiva. Endereço: Avenida Dr. Ruy Braga, S/N

Vila Mauriceia - Montes Claros

CEP: 39401-089 MG

E-mail: danielmestradounincor@yahoo.com.br 